Products and Services In Practice is provided to readers using text and images from the manufacturer, supplier or distributor and does not imply endorsement by $B D J$ In Practice. Normal and prudent research should be exercised before purchase or use of any product mentioned.

Please send product and services news through to David Westgarth, BDJ In Practice via: David.Westgarth@bda.org

\section{Brush up on your dental care}

Probiotics are gaining attention when it comes to gut health, but are emerging as a promising concept when it comes to maintaining oral health too. With a growing appetite from the general public to adopt simple strategies to nurture their health, probiotics for oral care deserve a place in your professional toolkit.

When it comes to the mouth, probiotics show potential to rebalance the oral microbiome, improving microbial-host dysbiosis and reducing the risk of dental disease. These beneficial bacteria can alter the ecology of our mouths, by crowding out and controlling levels of specific oral pathogens.

Luvbiotics, newly launched in the UK, has pioneered breakthrough bionet technology with the probiotic lactobacillus and xylitol with natural ingredients, which promotes good bacteria to balance oral flora and improve oral hygiene. The antimicrobial and anti-inflammatory effects of dental probiotics not only benefit the oral microbiome but also have further positive impact on the rest of the body. Just as good gut health affects the entire body, a balanced oral microbiome supports gut health and general immunity.

For more information visit www. luvbiotics.co.uk.

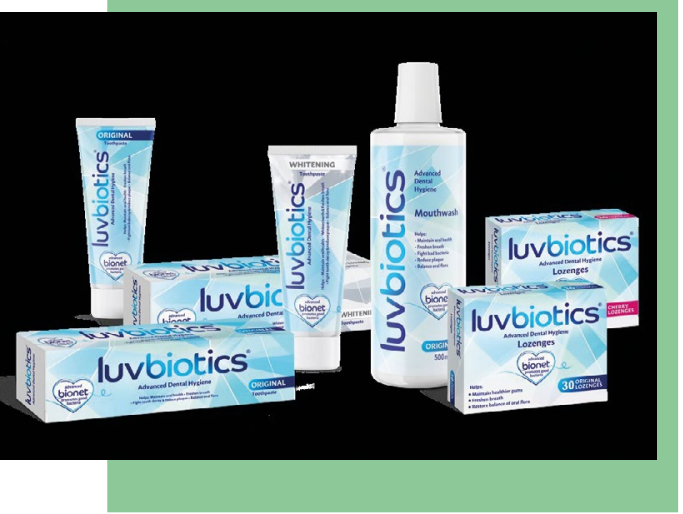

\title{
Gives patients the 'heads up'
}

Worn toothbrushes remove less plaque. With Oral-B power toothbrushes, it is a combination of the head and handle configuration, which work in perfect synergy, to give the user the ultimate

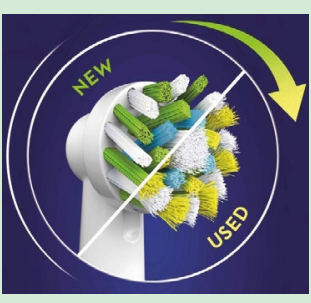
clean. The small round head mimics the action used in dental prophylaxis as it gently contours each tooth.
Convincing patients to change their toothbrush head regularly is not an easy task and can be a sensitive subject. Oral-B has launched a new brush head, called CleanMaximiser, which indicates when users should buy a replacement. Following dental professional recommendation, the bristles will change colour from green to yellow, after three months of use, indicating it's time for a new brush head.

The CleanMaximiser refills are compatible with all Oral-B models except the Pulsonic and iO.

\section{How dental defence can benefit the whole \\ dental team}

Following one of the most difficult and challenging periods in dentistry, the DDU is committed to supporting members. One way in which we do this is through our practice scheme, GROUPCARE, which is available to practices that have not incorporated, is free to open and provides support and savings for the whole dental team where at least half the dentists (minimum of two) in the practice are DDU members.

$\rightarrow$ When a dental practice joins the GROUPCARE scheme they have access to a valuable range of exclusive practice benefits including:

$\rightarrow 5 \%$ off DDU membership for all members in the practice

$\rightarrow$ An extra $50 \%$ saving on dental nurse membership

$\rightarrow$ Free practice manager membership

$\rightarrow$ A range of free CPD practice presentations, such as good record keeping, dealing with challenging patients, confidentiality and managing complaints

$\rightarrow$ Dedicated support from our team of dental liaison managers.
If all the dentists in a practice are DDU members, members can access additional benefits, including:

$\rightarrow 10 \%$ off DDU membership

$\rightarrow$ Free extended vicarious liability available to practice principals

$\rightarrow$ For practice principals, an additional benefit of DDU membership is access to free 24-hour employment law advice. Provided by Peninsula, the UK's leading employment law and health and safety consultancy providing business assistance to over 30,000 employers

$\rightarrow$ Access to free 24-hour health \& safety advice line also delivered through Peninsula.

GROUPCARE practices can view, update and administer their scheme details online via the DDU website.

To learn more about the benefits of the DDU's GROUPCARE scheme visit www.theddu.com/groupcare or follow @ the_ddu. 